PROCEEDINGS OF THE

AMERICAN MATHEMATICAL SOCIETY

Volume 137, Number 12, December 2009, Pages 3953-3967

S 0002-9939(09)10029-1

Article electronically published on July 30, 2009

\title{
JET SCHEMES OF THE COMMUTING MATRIX PAIRS SCHEME
}

\author{
B. A. SETHURAMAN AND KLEMEN ŠIVIC
}

(Communicated by Ted Chinburg)

\begin{abstract}
We show that for all $k \geq 1$ there exists an integer $N(k)$ such that for all $n \geq N(k)$ the $k$-th order jet scheme over the commuting $n \times n$ matrix pairs scheme is reducible.

At the other end of the spectrum, it is known that for all $k \geq 1$ the $k$-th order jet scheme over the commuting $2 \times 2$ matrices is irreducible; we show that the same holds for $n=3$.
\end{abstract}

\section{INTRODUCTION}

Recall that if $F$ is an algebraically closed field, $\left\{x_{i, j}, y_{i, j}, 1 \leq i, j \leq n\right\}$ are variables, $X=\left(x_{i, j}\right)$, and $Y=\left(y_{i, j}\right)$, the commuting $n \times n$ matrix pairs scheme $\mathcal{C}_{2, n}$ is $\operatorname{Spec}\left(F\left[\left\{x_{i, j}, y_{i, j}\right\}\right] / J\right)$, where $J$ is the ideal generated by the $n^{2}$ components of the matrix $X Y-Y X$. It is known that $\mathcal{C}_{2, n}$ is irreducible of dimension $n^{2}+n$ (see [11] or 2]), but it is an open problem if it is reduced.

For any scheme $X$ of finite type over $F$, we may define the scheme of $k$-th order jets $X^{(k)}$, whose closed points are in bijection with all morphisms $\operatorname{Spec}\left(F[t] / t^{k+1}\right) \rightarrow$ $X$ (see e.g. [10, §2]). When $X$ is affine, $X^{(k)}$ is also affine. In particular, for the affine scheme $\mathcal{C}_{2, n}, \mathcal{C}_{2, n}^{(k)}$ may be identified with $\operatorname{Spec}(R)$, where $R$ is defined as follows: Let $t, x_{i, j}^{(s)}$, and $y_{i, j}^{(s)}(0 \leq s \leq k, 1 \leq i, j \leq n)$ be new variables. For each generator $f_{u}$ of $J$, replace each $x_{i, j}$ and $y_{i, j}$ by $\sum_{s=0}^{k} x_{i, j}^{(s)} t^{s}$ and $\sum_{s=0}^{k} y_{i, j}^{(s)} t^{s}$ respectively, and expand $f_{u}$ as $\sum_{s=0}^{k} f_{u}^{(s)} t^{s} \bmod t^{k+1}$. Let $J^{(k)}$ be the ideal of $F\left[\left\{x_{i, j}^{(s)}, y_{i, j}^{(s)}\right\}\right]$ generated by $f_{u}^{(0)}, \ldots, f_{u}^{(k)}, u=1, \ldots, n^{2}$. Then $R=F\left[\left\{x_{i, j}^{(s)}, y_{i, j}^{(s)}\right\}\right] / J^{(k)}$. (Intuitively, the closed points of $\mathcal{C}_{2, n}^{(k)}$ yield paremeterized curves in $\mathbf{A}^{2 n^{2}}$ of degree $k$ that vanish to degree $k$ at closed points of $X$.)

Interest in jet schemes arises from the connections between the singularities of $X$ and the irreducibility of $X^{(k)}$. For instance, Mustaţă (9]) shows that for $X$ a locally complete intersection, $X$ has rational singularities if and only if all jet schemes $X^{(k)}$ are irreducible. Much earlier, Nash (12]) related the arc space of $X$ (the projective limit of the various $X^{(k)}$ ) to the exceptional divisors over the singular points of $X$ in a resolution of singularities.

Received by the editors November 4, 2008, and, in revised form, February 19, 2009.

2000 Mathematics Subject Classification. Primary 14M99.

The first author was supported by the National Science Foundation grant DMS-0700904.

The second author was supported by the Slovenian Research Agency.

(C)2009 American Mathematical Society 
Our interest in the specific jet schemes $\mathcal{C}_{2, n}^{(k)}$ arises from an open problem in commuting matrices. It is unknown whether the algebra $F[A, B, C]$ generated by three commuting $n \times n$ matrices, $A, B$, and $C$, has dimension bounded by $n$. (The corresponding answer is yes for algebras generated by two commuting matrices, and no for the algebra generated by four or more commuting matrices; see [4 for instance.) It is natural, while attacking this problem, to fix $C$ to be of various special forms, and to study the set of commuting pairs of matrices $(A, B)$ that lie in the centralizer of $C$. Write $J_{k+1}$ for the nilpotent Jordan block of size $k+1$. When $C$ has the special form consisting of $n$ copies of $J_{k+1}$ along the diagonal, the centralizer of $C$ in $M_{n(k+1)}(F)$ is isomorphic to $M_{n}(F[t]) / t^{k+1}=M_{n}(F)[t] / t^{k+1}$ (with $C$ corresponding to $t$ ), and the commuting pairs $(A, B)$ in the centralizer of $C$ are just pairs $A(t)=A_{0}+A_{1} t+\cdots+A_{k} t^{k}$ and $B(t)=B_{0}+B_{1} t+\cdots+B_{k} t^{k}$ that commute in $M_{n}(F)[t] / t^{k+1}$.

(This is elementary and can be gleaned from the Toeplitz structure of the matrices that commute with $C$; see e.g. [1]. Any matrix $A \in M_{n(k+1)}(F)$ that commutes with $C$ is of the block form $\left(A_{i, j}\right)$, where each $A_{i, j}$ is a $(k+1) \times(k+1)$ matrix of the form

$$
\left(\begin{array}{ccccc}
x_{i, j}^{(0)} & x_{i, j}^{(1)} & x_{i, j}^{(2)} & \ldots & x_{i, j}^{(k)} \\
0 & x_{i, j}^{(0)} & x_{i, j}^{(1)} & x_{i, j}^{(2)} & \vdots \\
\vdots & \ddots & \ddots & \ddots & \ddots \\
\vdots & \ddots & \ddots & \ddots & x_{i, j}^{(1)} \\
0 & \ldots & \ldots & 0 & x_{i, j}^{(0)}
\end{array}\right) .
$$

The $n \times n$ matrix $A_{0}$ is defined as $\left(x_{i, j}^{(0)}\right), A_{1}$ as $\left(x_{i, j}^{(1)}\right)$, etc. We will routinely identify the centralizer of $C$ in $M_{n(k+1)}(F)$ with $M_{n}(F)[t] / t^{k+1}$.)

It follows by expanding the commutator $[A(t), B(t)]$ in powers of $t$ that the equations for $A(t)$ and $B(t)$ to commute are just the generators of $J^{(k)}$ described above. Hence, the commuting pairs in the centralizer of $C$ correspond to the closed points of $\mathcal{C}_{2, n}^{(k)}$, as $F$ is algebraically closed. Irreducibility of $\mathcal{C}_{2, n}^{(k)}$ would show that for all commuting triples of $n(k+1) \times n(k+1)$ matrices $(A, B, C)$ with $C$ having Jordan form equal to $n$ copies of $J_{k+1}$ up to addition of scalars, the dimension of $F[A, B, C]$ would be bounded by $n(k+1)$. (See Corollary 4.7 below for the $n=3$ case, for example.) It is thus of interest to know if $\mathcal{C}_{2, n}^{(k)}$ is irreducible.

The goal of this paper is to show that the answer to the question is negative in general: we prove that for $k \geq 1$, there exists an integer $N(k)$ such that for all $n \geq N(k), \mathcal{C}_{2, n}^{(k)}$ is reducible. At the other end of the spectrum, we also show that $\mathcal{C}_{2, n}^{(k)}$ is irreducible for $n=3$ and all $k \geq 1$. (It is known-see [14]-that $\mathcal{C}_{2, n}^{(k)}$ is irreducible for $n=2$ and all $k \geq 1$.)

We note in passing that the results of 14 showed that for $n=2, \mathcal{C}_{2, n}^{(k)}$ is very naturally related to jet schemes over determinantal varieties of rank at most 1 , which then led the first named author along with Tomaž Košir to study jet schemes over determinantal varieties in general ([6], 7]; see also [5]). These were also studied independently by Cornelia Yuen in her thesis ([15]).

This paper is a direct result of collaboration between the authors during the 5th Linear Algebra Workshop held at Kranjska Gora, Slovenia, in 2008. The authors wish to thank the organizers for an enjoyable and very productive meeting. 


\section{A Distinguished OPEn Set OF $\mathcal{C}_{2, n}^{(k)}$}

Consider the open subscheme $\mathcal{U}$ defined by the condition that $A_{0}=\left(x_{i, j}^{(0)}\right)$ is 1-regular. (Recall that the matrix $A_{0}$ is $r$-regular if all eigenspaces of $A_{0}$ are of dimension at most $r$; this is an open set condition. See [14. Prop. 1]. 1-regular matrices in $M_{n}(F)$ can be characterized by several equivalent conditions, including: (i) $\operatorname{dim}_{F}\left(F\left[A_{0}\right]\right)=n$, and (ii) the centralizer of $F\left[A_{0}\right]$ in $M_{n}(F)$ is precisely $F\left[A_{0}\right]$.) The goal of this section is to prove that $\mathcal{U}$ is irreducible, of dimension $\left(n^{2}+n\right)(k+1)$.

We will give two proofs of this result. The first proof involves a characterization of matrices $A(t)=A_{0}+A_{1} t+\cdots+A_{k} t^{k}$ such that $A_{0}$ is 1-regular in terms of the algebra generated by $A(t)$ and $t$. The second proof invokes general results about jet schemes over smooth schemes and uses a Jacobian computation in [13]. Once $\mathcal{U}$ is known to be irreducible, we can argue that if $\mathcal{C}_{2, n}^{(k)}$ were irreducible it should equal the closure of $\mathcal{U}$, so it should also have dimension $\left(n^{2}+n\right)(k+1)$. In Section 3 ahead, we will use this expected dimension to show that $\mathcal{C}_{2, n}^{(k)}$ is reducible for large enough $n$.

2.1. A characterization of matrices $A(t)$ with $A_{0}$ 1-regular. We begin with the first proof that $\mathcal{U}$ is irreducible of the stated dimension. Let $\mathcal{V}\left(\mathcal{C}_{2, n}^{(k)}\right)$ denote the closed points of $\mathcal{C}_{2, n}^{(k)}$, which, since $F$ is algebraically closed, is just the algebraic set in $\mathbf{A}^{2 n^{2}(k+1)}$ consisting of matrices $A(t)=A_{0}+A_{1} t+\cdots+A_{k} t^{k}$ and $B(t)=$ $B_{0}+B_{1} t+\cdots+B_{k} t^{k}$ that commute in $M_{n}(F)[t] / t^{k+1}$. Similarly, let $U=\mathcal{U} \cap \mathcal{V}\left(\mathcal{C}_{2, n}^{(k)}\right)$ be the set of closed points of $\mathcal{U}$ ([8, Chap. 2, Lemma 4.3]), so $U$ consists of those commuting pairs above in which $A_{0}$ is 1-regular. Note that $\mathcal{V}\left(\mathcal{C}_{2, n}^{(k)}\right)$ is dense in $\mathcal{C}_{2, n}^{(k)}$ (see e.g. [8, Chap. 2, Remark 3.49]). Using the fact that every prime ideal of $R$ (where $R$ is as in Section 1) is an intersection of maximal ideals, it is easy to see that $U$ is dense in $\mathcal{U}$ and that the dimension of $U$ as an algebraic set is the same as the dimension of $\mathcal{U}$. We will hence work with $\mathcal{V}\left(\mathcal{C}_{2, n}^{(k)}\right)$ and $U$. The goal in this subsection is to prove the irreducibility of $U$ by deriving a characterization of matrices $A(t)=A_{0}+A_{1} t+\cdots+A_{k} t^{k}$ such that $A_{0}$ is 1-regular in terms of the algebra generated by $A(t)$ and $t$.

Let $z_{1}, \ldots, z_{k}$ be noncommuting variables. Given $n_{1}$ copies of $z_{1}, \ldots, n_{k}$ copies of $z_{k}$, we will denote by $S\left(z_{1}^{\left[n_{1}\right]}, \ldots, z_{k}^{\left[n_{k}\right]}\right)$ the sum of all noncommuting monomials of degree $n_{1}$ in $z_{1}, \ldots, n_{k}$ in $z_{k}$. For instance, $S\left(x^{[2]}, y^{[1]}\right)=x^{2} y+x y x+y x^{2}$.

We will find it convenient to also adopt the following convention: given the not necessarily distinct noncommutative variables $y_{1}, \ldots, y_{t}$, of which $n_{1}$ are all equal (to say $y_{i_{1}}$ ), $\ldots, n_{k}$ are all equal (to say $y_{i_{k}}$ ), $n_{1}+\cdots+n_{k}=t$, the expression $S\left(y_{1}, \ldots, y_{t}\right)$ will denote the polynomial $S\left(y_{i_{1}}^{\left[n_{1}\right]}, \ldots, y_{i_{k}}^{\left[n_{k}\right]}\right)$ defined above. Notice that if $y_{\sigma(1)}, \ldots, y_{\sigma(t)}$ is any rearrangement of the list $y_{1}, \ldots, y_{t}$, then $S\left(y_{1}, \ldots, y_{t}\right)=S\left(y_{\sigma(1)}, \ldots, y_{\sigma(t)}\right)$ just by definition.

Expressions such as $S\left(x^{[d]}, y_{1}, \ldots, y_{t}\right)$, with $d>0$ and $y_{1}, \ldots, y_{t}$ not necessarily distinct among themselves but distinct from $x$, are similarly defined. We define $S\left(x^{[0]}, y_{1}, \ldots, y_{t}\right)$ to be $S\left(y_{1}, \ldots, y_{t}\right)$ and $S\left(x^{[d]}, y_{1}, \ldots, y_{t}\right)$ to be zero if $d<0$.

Let $q(x)=\sum_{j=0}^{N-1} c_{j} x^{j}$ be a polynomial with coefficients in $F$ in the variable $x$. Given the not necessarily distinct noncommutative variables $y_{1}, \ldots, y_{t}$, 
none of which commutes with $x$, we let $d_{y_{1}, \ldots, y_{t}}(q(x))$ denote the (noncommutative) polynomial $\sum_{j=0}^{N-1} c_{j} S\left(x^{[j-t]}, y_{1}, \ldots, y_{t}\right)$ (recall from above the convention for $S\left(x^{[j-t]}, y_{1}, \ldots, y_{t}\right)$ if $\left.j-t \leq 0\right)$.

Note that the polynomial $d_{y_{1}, \ldots, y_{t}}(q(x))$ is symmetric with respect to the permutation of the $y_{j}$ because $S$ is symmetric with respect to the permutation of the $y_{j}$.

Given matrices $A_{0}, \ldots, A_{k}$, an expression such as $d_{A_{i_{1}} \ldots A_{i_{r}}}\left(q_{j}\left(A_{0}\right)\right)$ indicates the following: Take the polynomial $d_{y_{i_{1}} \ldots y_{i_{r}}}\left(q_{j}(x)\right)$ defined above for the noncommuting variables $x, y_{i_{1}}, \ldots, y_{i_{r}}$ and the polynomial $q_{j}(x) \in F[x]$, and evaluate it at $x=A_{0}$ and $y_{i_{j}}=A_{i_{j}}$.

We begin with the following lemma, which will go into our characterization:

Lemma 2.1. Given $N>0$ and $N(k+1)$ elements $c_{i, j} \in F$ for $0 \leq i<N$, $0 \leq j \leq k$, define the polynomials $q_{j}(x), 0 \leq j \leq k$, by $q_{j}(x)=\sum_{i=0}^{N-1} c_{i, j} x^{i}$. Given $A(t)=A_{0}+A_{1} t+\cdots+A_{k} t^{k}$ in $M_{n}(F)[t] / t^{k+1}$, we have

$$
\sum_{j=0}^{k} \sum_{i=0}^{N-1} c_{i, j}(A(t))^{i} t^{j}=B_{0}+B_{1} t+\cdots+B_{k} t^{k},
$$

where $B_{0}=q_{0}\left(A_{0}\right)$, and more generally, for $s=1, \ldots, k$,

$$
B_{s}=\sum_{j=0}^{s-1}\left(\sum_{r=1}^{s-j} \sum_{\substack{i_{1} \geq i_{2} \geq \ldots \geq i_{r}>0 \\ i_{1}+\ldots+i_{r}=s-j}} d_{A_{i_{1}} \ldots A_{i_{r}}}\left(q_{j}\left(A_{0}\right)\right)\right)+q_{s}\left(A_{0}\right) .
$$

Proof. We first consider a single power $(A(t))^{i}=\left(A_{0}+A_{1} t+\cdots+A_{k} t^{k}\right)^{i}$. Expanding the parenthesis, we may write this as $\sum_{l=0}^{k} G_{l}^{(i)} t^{l}$, where $G_{0}^{(i)}=\left(A_{0}\right)^{i}$, and for $l \geq 1$,

$$
G_{l}^{(i)}=\sum_{r=1}^{l} \sum_{\substack{i_{1} \geq i_{2} \geq \cdots \geq i_{r}>0 \\ i_{1}+\cdots+i_{r}=l}} S\left(A_{0}^{[i-r]}, A_{i_{1}}, \ldots, A_{i_{r}}\right) .
$$

We thus write

$$
\sum_{j=0}^{k} \sum_{i=0}^{N-1} c_{i, j}(A(t))^{i} t^{j}=\sum_{j=0}^{k} \sum_{i=0}^{N-1} c_{i, j}\left(G_{0}^{(i)}+G_{1}^{(i)} t+\cdots+G_{k}^{(i)} t^{k}\right) t^{j} .
$$

We wish to rewrite the right side of the equation above as $B_{0}+B_{1} t+\cdots+B_{k} t^{k}$. Now $B_{0}$ is the coefficient of $t^{0}$ on the right side of the equation above, which is simply $\sum_{i=0}^{N-1} c_{i, 0} G_{0}^{(i)}$. Since $G_{0}^{(i)}=\left(A_{0}\right)^{i}$, we find $B_{0}=q_{0}\left(A_{0}\right)$, with $q_{0}$ as in the statement of the theorem.

Similarly, for $s \geq 1, B_{s}$ is the coefficient of $t^{s}$ on the right side of equation (2.4), so

$$
B_{s}=\sum_{j=0}^{s} \sum_{i=0}^{N-1} c_{i, j} G_{s-j}^{(i)} .
$$

When $j=s$, the inner term on the right side of the equation above is $\sum_{i=0}^{N-1} c_{i, s} G_{0}^{(i)}$ $=\sum_{i=0}^{N-1} c_{i, s}\left(A_{0}\right)^{i}=q_{s}\left(A_{0}\right)$. 
For a fixed $j=j^{*}<s$, the the inner term on the right side of equation (2.5) equals, from equation (2.3) above,

$$
\begin{array}{r}
\sum_{i=0}^{N-1} c_{i, j^{*}}\left[\sum_{r=1}^{s-j^{*}} \sum_{\substack{i_{1} \geq i_{2} \geq \cdots \geq i_{r}>0 \\
i_{1}+\cdots+i_{r}=s-j^{*}}} S\left(A_{0}^{[i-r]}, A_{i_{1}}, \ldots, A_{i_{r}}\right)\right] \\
=\sum_{r=1}^{s-j^{*}} \sum_{\substack{i_{1} \geq i_{2} \geq \cdots \geq i_{r}>0 \\
i_{1}+\ldots+i_{r}=s-j^{*}}} d_{A_{i_{1}} \ldots A_{i_{r}}}\left(q_{j^{*}}\left(A_{0}\right)\right) .
\end{array}
$$

Adding together these expressions for $j=s, s-1, \ldots, 0$, we find that $B_{s}$ is indeed as described in the statement of the theorem.

We now prove the following:

Theorem 2.2. Let $A=A(t)=A_{0}+A_{1} t+\cdots+A_{k} t^{k}$ be given in $M_{n}(F)[t] / t^{k+1} \subset$ $M_{n(k+1)}(F)$. Then the following are equivalent:

(1) $A_{0}$ is 1-regular.

(2) The $n(k+1)$ elements $(A(t))^{i} t^{j}, 0 \leq i<n, 0 \leq j \leq k$, in $F[A, t]$ are $F$-linearly independent.

(3) $F[A, t]$ has dimension $n(k+1)$ as an $F$-subalgebra of $M_{n}(F)[t] / t^{k+1}$ (and hence of $\left.M_{n(k+1)}(F)\right)$.

(4) Any $B=B(t)$ that commutes with both $A(t)$ and $t$ is for the form $B(t)=$ $\sum_{j=0}^{k} \sum_{i=0}^{n-1} c_{i, j}(A(t))^{i} t^{j}$ for arbitrary elements $c_{i, j} \in F, 0 \leq i<n, 0 \leq$ $j \leq k$.

(5) Any $B=B(t)$ that commutes with both $A(t)$ and $t$ is for the form $B(t)=$ $B_{0}+B_{1} t+\cdots+B_{k} t^{k}$, where $B_{0}=q_{0}\left(A_{0}\right)$, and more generally, for $s=$ $1, \ldots, k$,

$$
B_{s}=\sum_{j=0}^{s-1}\left(\sum_{r=1}^{s-j} \sum_{\substack{i_{1} \geq i_{2} \geq \cdots \geq i_{r}>0 \\ i_{1}+\ldots+i_{r}=s-j}} d_{A_{i_{1}} \ldots A_{i_{r}}}\left(q_{j}\left(A_{0}\right)\right)\right)+q_{s}\left(A_{0}\right),
$$

where $q_{0}, q_{1}, \ldots, q_{k}$ are arbitrary polynomials with coefficients in $F$ of degree at most $n-1$.

Proof. Note that (4) $\Leftrightarrow$ (5) is an immediate consequence of Lemma 2.1, with the $N$ of that lemma replaced by $n$.

(11) $\Rightarrow$ (2): Since $A_{0}$ is 1 -regular, the matrices $I_{n}, A_{0}, \ldots,\left(A_{0}\right)^{n-1}$ are $F$ linearly independent in $M_{n}(F)$. It follows immediately, by sequentially considering the coefficients of $t^{0}, t^{1}, \ldots, t^{k}$ in the left hand side of an equation such as

$$
\sum_{j=0}^{k} \sum_{i=0}^{n-1} c_{i, j}(A(t))^{i} t^{j}=0
$$

for arbitrary elements $c_{i, j} \in F$, that the $n(k+1)$ elements $(A(t))^{i} t^{j}, 0 \leq i<n$, $0 \leq j \leq k$, must be $F$-linearly independent.

(2) $\Rightarrow$ (3): This implication is clear since the algebra generated by two commuting matrices in $M_{n(k+1)}(F)$ has dimension at most $n(k+1)$ by classical results (see [4] for example). 
(3) $\Rightarrow$ (11): Let $A^{\prime}=A_{0}+A_{1} t+\cdots+A_{k} t^{k}+0 t^{k+1}+\cdots+0 t^{k+l} \in M_{n}(F)[t] / t^{k+l+1}$, where $l \geq 0$ is yet to be determined. (Note that $M_{n}(F)[t] / t^{k+l+1} \subset M_{n(k+l+1)}(F)$, with $t$ corresponding to the $n(k+l+1) \times n(k+l+1)$ matrix consisting of $n$ Jordan blocks of size $k+l+1$ along the diagonal.) Write $E$ for the algebra $F[A, t] \subset$ $M_{n}(F)[t] / t^{k+1}$, and $E^{\prime}$ for the algebra $F\left[A^{\prime}, t\right] \subset M_{n}(F)[t] / t^{k+l+1}$. Given a nonzero element $B=B_{i} t^{i}+B_{i+1} t^{i+1}+\cdots$ in $M_{n}(F)[t] / t^{k+1}$ with $B_{i} \neq 0$, we define the degree of $B$ to be $i$; we also define the degree of 0 to be infinity. We have the filtration by $F$-spaces $E=E_{0} \supset E_{1} \supset \cdots \supset E_{k} \supset E_{k+1}=0$, where $E_{i}$ consists of those elements in $E$ with degree $i$ or higher. We have a similar filtration $E^{\prime}=E_{0}^{\prime} \supset E_{1}^{\prime} \supset \cdots \supset E_{k+l}^{\prime} \supset E_{k+l+1}^{\prime}=0$. The map $\pi: M_{n}(F)[t] / t^{k+l+1} \rightarrow$ $M_{n}(F)[t] / t^{k+1}$ that maps the element $B_{0}+B_{1} t+\cdots+B_{k} t^{k}+B_{k+1} t^{k+1}+\cdots+$ $B_{k+l} t^{k+l}$ to $B_{0}+B_{1} t+\cdots+B_{k} t^{k}$ is an $F$-algebra homomorphism and in particular an $F$ vector space homomorphism. Equation (2.2) in Lemma 2.1 (applied with $k+l$ substituted for $k$ ) shows that the coefficient of $t^{s}$ of a polynomial expression $f\left(A^{\prime}, t\right)$ depends only on the coefficients of $t^{0}, t^{1}, \ldots, t^{s}$ in $A^{\prime}$. More specifically, if $f\left(A^{\prime}, t\right)=B_{i} t^{i}+B_{i+1} t^{i+1}+\cdots+B_{k} t^{k}+B_{k+1} t^{k+1}+\cdots+B_{k+l} t^{k+l} \bmod t^{k+l+1}$, then $f(A, t)=B_{i} t^{i}+B_{i+1} t^{i+1}+\cdots+B_{k} t^{k} \bmod t^{k+1}$. Thus, $\pi\left(f\left(A^{\prime}, t\right)\right)=f(A, t)$, so $\pi$ maps each $E_{i}^{\prime}$ surjectively to $E_{i}$ for $i=0, \ldots, k$. Moreover, the kernel of the induced $F$ vector space map $E_{i}^{\prime} \mapsto E_{i} \mapsto E_{i} / E_{i+1}$ is precisely $E_{i+1}^{\prime}$. We thus have $F$ vector space isomorphisms between $E_{i}^{\prime} / E_{i+1}^{\prime}$ and $E_{i} / E_{i+1}$ for $i=0, \ldots, k$.

Now assume that $F[A, t]$ has dimension $n(k+1)$ but that $A_{0}$ is not 1-regular. If we write a polynomial expression $f(A, t)$ as $B_{0}+B_{1} t+\cdots+B_{k} t^{k}$, Lemma 2.1 tells us that $B_{0}=q_{0}\left(A_{0}\right)$, for a suitable polynomial $q_{0}$. Conversely, given a polynomial $q_{0} \in F[x], q_{0}(A)=q_{0}\left(A_{0}\right)+t(\ldots)$. It follows that $E_{0} / E_{1} \cong F\left[A_{0}\right]$. Since $A_{0}$ not being 1-regular means that $\operatorname{dim}_{F} F\left[A_{0}\right]<n$, we find $\operatorname{dim}_{F} E_{0} / E_{1}<n$. The fact that $\operatorname{dim}_{F} E=n(k+1)$ and that $\operatorname{dim}_{F} E=\sum_{i=0}^{k} \operatorname{dim}_{F}\left(E_{i} / E_{i+1}\right)$ shows that $\operatorname{dim}_{F}\left(E_{i} / E_{i+1}\right)>n$ for some $i$ with $k \geq i \geq 1$. It follows from the isomorphism above that $\operatorname{dim}_{F} E_{i}^{\prime} / E_{i+1}^{\prime}>n$. But for any $j<k+l$, we have an injective $F$ vector space map from $E_{j}^{\prime} / E_{j+1}^{\prime}$ to $E_{j+1}^{\prime} / E_{j+2}^{\prime}$ that sends the class of an element $B_{j} t^{j}+B_{j+1} t^{j+1}+\cdots$ to the class of the element $B_{j} t^{j+1}+B_{j+1} t^{j+2}+\cdots$. Hence $\operatorname{dim}_{F} E_{j}^{\prime} / E_{j+1}^{\prime}>n$ for all $j$ with $k+l \geq j \geq i$. It is clear that by taking $l$ large enough, we can make $\operatorname{dim}_{F} E^{\prime}=\sum_{i=0}^{k+l} \operatorname{dim}_{F} E_{i}^{\prime} / E_{i+1}^{\prime}>n(k+l+1)$, no matter what the values of $\operatorname{dim}_{F} E_{s}^{\prime} / E_{s+1}^{\prime}$ are for $s<i$. But this violates the classical result that the dimension of the $F$-algebra generated by the commuting matrices $A^{\prime}$ and $t$ in $M_{n}(F)[t] / t^{k+l+1} \subset M_{n(k+l+1)}(F)$ is bounded by $n(k+l+1)$. Hence, $A_{0}$ must be 1-regular.

(3) $\Leftrightarrow$ (4): Both implications follow from [13, Theorem 1.1], which states that $F[A, t] \subseteq M_{n(k+1)}(F)$ has dimension $n(k+1)$ iff the centralizer of $F[A, t]$ in $M_{n(k+1)}(F)$ is $F[A, t]$ itself, along with part (2) above of this theorem.

We have the following corollary immediately:

Corollary 2.3. The sets $U \subset \mathcal{V}\left(\mathcal{C}_{2, n}^{(k)}\right)$ and $\mathcal{U} \subset \mathcal{C}_{2, n}^{(k)}$ are irreducible, of dimension $\left(n^{2}+n\right)(k+1)$.

Proof. Viewed as algebraic sets, the set of all $A(t)$ with $A_{0}$ 1-regular is an open set in $\mathbf{A}^{n^{2}(k+1)}$, since $A_{0}$ is constrained to live in the open set of $\mathbf{A}^{n^{2}}$ of 1-regular matrices while $A_{1}, \ldots, A_{k}$ can be arbitrary. Theorem 2.2. part (4), above shows that the set of matrices $B(t)$ that commute with $A(t)$ is defined by the $n(k+1)$ arbitrary 
elements $c_{i, j} \in F$. Hence $U$, which is the set of commuting pairs $(A(t), B(t))$ with $A_{0}$ 1-regular, is isomorphic as an algebraic set to a product of an open set of $\mathbf{A}^{n^{2}(k+1)}$ and $\mathbf{A}^{n(k+1)}$. The irreducibility and dimension of $U$ immediately follow. The same results hold for $\mathcal{U}$ as described at the beginning of this subsection.

The theorem yields another corollary:

Corollary 2.4. Let $A(t)=A_{0}+A_{1} t+\cdots+A_{k} t^{k}$ and $B(t)=B_{0}+B_{1} t+\cdots+B_{k} t^{k}$ commute in $M_{n}(F)[t] / t^{k+1}$, and assume that $A_{0}$ is 1-regular. Then for any $A_{k+1} \in$ $M_{n}(F)$, there exists $B_{k+1} \in M_{n}(F)$ such that $A^{\prime}(t)=A_{0}+A_{1} t+\cdots+A_{k} t^{k}+$ $A_{k+1} t^{k+1}$ and $B(t)=B_{0}+B_{1} t+\cdots+B_{k} t^{k}+B_{k+1} t^{k+1}$ commute in $M_{n}(F)[t] / t^{k+2}$. In particular, the map $\pi_{k+1}: \mathcal{V}\left(\mathcal{C}_{2, n}^{(k+1)}\right) \rightarrow \mathcal{V}\left(\mathcal{C}_{2, n}^{(k)}\right)$ that sends a general pair $\left(A_{0}+\right.$ $\left.A_{1} t+\cdots+A_{k} t^{k}+A_{k+1} t^{k+1}, B_{0}+B_{1} t+\cdots+B_{k} t^{k}+B_{k+1} t^{k+1}\right)$ to $\left(A_{0}+A_{1} t+\right.$ $\left.\cdots+A_{k} t^{k}, B_{0}+B_{1} t+\cdots+B_{k} t^{k}\right)$ is surjective when restricted to the open sets of $\mathcal{V}\left(\mathcal{C}_{2, n}^{(k+1)}\right)$ and $\mathcal{V}\left(\mathcal{C}_{2, n}^{(k)}\right)$ where $A_{0}$ is 1-regular.

Proof. This is immediate from Theorem 2.2. part (5), where we see that the solution for $B_{s}(s=0, \ldots, k)$ depends only on $A_{i}(i=0, \ldots, s)$ and that having solved for $B_{s}$, one can solve for $B_{s+1}$ given any $A_{s+1}$.

Remark 2.5. Note that $\pi_{1}: \mathcal{C}_{2, n}^{(1)} \rightarrow \mathcal{C}_{2, n}$ is trivially surjective since given $A_{0}$ and $B_{0}$ in $M_{n}(F)$ that commute, the matrices $A_{0}+0 \cdot t$ and $B_{0}+0 \cdot t$ commute in $M_{n}(F)[t] / t^{2}$. However, this simple extension to a higher degree in $t$ fails for $k>1$. For instance, let $X$ and $Y$ be any two matrices in $M_{n}(F)$ that do not commute with each other. Then $A=0+X t$ and $B=0+Y t$ commute in $M_{n}(F)[t] / t^{2}$, but it is clear by writing the equation for the $t^{2}$ component that $A^{\prime}=0+X t+P t^{2}$ and $B^{\prime}=0+Y t+Q t^{2}$ cannot commute in $M_{n}(F)[t] / t^{3}$ for any choice of $P$ and $Q$.

We also have the following:

Corollary 2.6. Given the commuting pair $A(t)=A_{0}+A_{1} t+\cdots+A_{k} t^{k}$ and $B(t)=B_{0}+B_{1} t+\cdots+B_{k} t^{k}$ in $M_{n}(F)[t] / t^{k+1}$, with $A_{0}$ 1-regular, we have:

$$
\left[A_{1}, B_{k}\right]+\left[A_{2}, B_{k-1}\right]+\cdots+\left[A_{k}, B_{1}\right] \in \operatorname{Ad}\left(A_{0}\right) .
$$

Proof. Taking $A_{k+1}=0$, Corollary 2.4 shows that we can find $B_{k+1}$ such that $A_{0}+A_{1} t+\cdots+A_{k} t^{k}+0 \cdot t^{k+1}$ and $B(t)=B_{0}+B_{1} t+\cdots+B_{k} t^{k}+B_{k+1} t^{k+1}$ commute in $M_{n}(F)[t] / t^{k+2}$. Writing the equation for the matrices in $t^{k+1}$, we find

$$
\left[A_{0}, B_{k+1}\right]+\left[A_{1}, B_{k}\right]+\left[A_{2}, B_{k-1}\right]+\cdots+\left[A_{k}, B_{1}\right]=0 .
$$

Since the first term is in $A d\left(A_{0}\right)$, the sum of the remaining terms must also be in $\operatorname{Ad}\left(A_{0}\right)$.

Remark 2.7. Note that the proofs of Theorem 2.2 and of Corollaries 2.4 and 2.6 did not depend on $F$ being algebraically closed.

2.2. Alternative proof of Corollary 2.3. For the second proof that $\mathcal{U}$ is irreducible of dimension $\left(n^{2}+n\right)(k+1)$, we recall the following: if $X$ is a smooth scheme of finite type over $F$ of dimension $d$, then $X^{(k)}$ is an $\mathbf{A}^{d k}$ bundle over $X$. 
See [10, §2] for instance. The heart of the result is that for a smooth scheme of finite type over $F$ of dimension $d$, there exists an open cover $U_{\alpha}$ with étale maps $g_{\alpha}: U_{\alpha} \rightarrow \mathbf{A}^{d}$ (see e.g. [8, §6.2.2]), and since $g_{\alpha}$ is étale, $U_{\alpha}^{(k)} \cong\left(\mathbf{A}^{d}\right)^{(k)} \times{ }_{\mathbf{A}^{d}} U_{\alpha}$.

Recall that since $F$ is algebraically closed, smoothness and regularity coincide (see [8, Chap. 4, Definition 3.28]). In [13, Theorem 1.1], the authors use the Jacobian criterion to show that a closed point $\left(A_{0}, B_{0}\right)$ of the commuting matrices scheme $\mathcal{C}_{2, n}$ is regular if and only if $\operatorname{dim}_{F}\left[A_{0}, B_{0}\right]=n$. (Note that the Jacobian criterion in [13] is applied to the ideal $J$ defined in Section 1, which is not known to be radical, and hence the result of $[13$, Theorem 1.1, Part 4] really applies to the commuting matrices scheme and not necessarily the commuting matrices variety in the sense used in 13.) When $A_{0}$ is 1-regular, then the two characterizations described at the beginning of Section 2 show that $\operatorname{dim}_{F}\left[A_{0}, B_{0}\right]=n$ for any $B_{0}$ that commutes with $A_{0}$. Thus, letting $\mathcal{W}$ denote the open subscheme of $\mathcal{C}_{2, n}$ where $A_{0}$ is 1-regular and $W$ the set of closed points of $\mathcal{W}$, we find that the points of $W$ are all nonsingular, and hence (see [8, Chap. 4, Corollary 2.17] for instance), $\mathcal{W}$ is smooth. Thus, $\mathcal{U}$, which is $\mathcal{W}^{(k)}$ (as $\mathcal{W} \rightarrow \mathcal{C}_{2, n}$ is étale), is simply an $\mathbf{A}^{\left(n^{2}+n\right) k}$ bundle over $\mathcal{W}$. Being an open set of $\mathcal{C}_{2, n}, \mathcal{W}$ is irreducible of dimension $n^{2}+n$, so $\mathcal{U}$ is irreducible of dimension $\left(n^{2}+n\right)(k+1)$.

\section{REDUCIBILITy OF $\mathcal{C}_{2, n}^{(k)}$ FOR LARGE $n$}

We prove in this section that for any $k$ and large enough $n, \mathcal{C}_{2, n}^{(k)}$ is reducible. We show that for each $k \geq 1$, there exists an integer $N(k)$ such that for all $n \geq N(k)$, $\mathcal{V}\left(\mathcal{C}_{2, n}^{(k)}\right)$ has a closed set of dimension greater than $\left(n^{2}+n\right)(k+1)$. Since the open set $U$ considered in the previous section is irreducible of dimension $\left(n^{2}+n\right)(k+1)$, $\mathcal{V}\left(\mathcal{C}_{2, n}^{(k)}\right)$ cannot be irreducible for such $n$, and hence $\mathcal{C}_{2, n}^{(k)}$ cannot be irreducible for such $n$.

Theorem 3.1. For each $k \geq 1$, there exists an integer $N(k)$ such that for all $n \geq N(k), \mathcal{V}\left(\mathcal{C}_{2, n}^{(k)}\right)$, and hence $\mathcal{C}_{2, n}^{(k)}$, is reducible.

Proof. Let $n=3 a+b$, and write $n \times n$ matrices as $4 \times 4$ block matrices where the first 3 rows and columns are of dimension $a$ and the last ones are of dimension $b$. Let $W$ be the closed set of all pairs $(A(t), B(t)) \in \mathcal{V}\left(\mathcal{C}_{2, n}^{(k)}\right)$ such that in block form

$$
\begin{gathered}
A_{0}=\left(\begin{array}{llll}
0 & I & 0 & 0 \\
0 & 0 & I & 0 \\
0 & 0 & 0 & 0 \\
0 & 0 & 0 & 0
\end{array}\right), B_{0}=\left(\begin{array}{cccc}
0 & B_{1}^{(0)} & B_{2}^{(0)} & B_{3}^{(0)} \\
0 & 0 & B_{1}^{(0)} & 0 \\
0 & 0 & 0 & 0 \\
0 & 0 & B_{4}^{(0)} & 0
\end{array}\right), \\
A_{1}=\left(\begin{array}{cccc}
A_{11}^{(1)} & A_{12}^{(1)} & A_{13}^{(1)} & A_{14}^{(1)} \\
A_{21}^{(1)} & A_{22}^{(1)} & A_{23}^{(1)} & A_{24}^{(1)} \\
0 & A_{32}^{(1)} & A_{33}^{(1)} & A_{34}^{(1)} \\
A_{41}^{(1)} & A_{42}^{(1)} & A_{43}^{(1)} & A_{44}^{(1)}
\end{array}\right), B_{1}=\left(\begin{array}{cccc}
B_{11}^{(1)} & B_{12}^{(1)} & B_{13}^{(1)} & B_{14}^{(1)} \\
B_{21}^{(1)} & B_{22}^{(1)} & B_{23}^{(1)} & B_{24}^{(1)} \\
0 & B_{32}^{(1)} & B_{33}^{(1)} & B_{34}^{(1)} \\
B_{41}^{(1)} & B_{42}^{(1)} & B_{43}^{(1)} & B_{44}^{(1)}
\end{array}\right),
\end{gathered}
$$


and $A_{2}, \ldots, A_{k}, B_{2}, \ldots, B_{k} \in M_{n}(F)$ are arbitrary such that $[A(t), B(t)]=0$. The commutativity relation of $A(t)$ and $B(t)$ is given by

$$
\begin{aligned}
{\left[A_{0}, B_{0}\right] } & =0, \\
{\left[A_{0}, B_{1}\right]+\left[A_{1}, B_{0}\right] } & =0, \\
{\left[A_{0}, B_{2}\right]+\left[A_{1}, B_{1}\right]+\left[A_{2}, B_{0}\right] } & =0, \\
\vdots & =\vdots \\
{\left[A_{0}, B_{k}\right]+\left[A_{1}, B_{k-1}\right]+\cdots+\left[A_{k}, B_{0}\right] } & =0 .
\end{aligned}
$$

The first equation is automatically satisfied since $B_{0}$ has been chosen to commute with $A_{0}$. As for the second equation, note that $\left[A_{0}, B_{1}\right]+\left[A_{1}, B_{0}\right]$ has zeros in the entries $(2,1),(3,1),(4,1),(3,2)$, and $(3,4)$, so the second equation in the system of equations (3.1) can be described by $6 a^{2}+4 a b+b^{2}-1$ equations (the subtraction of 1 is because the trace of $[X, Y]$ is zero, so the diagonal entries are always dependent). Each of the remaining equations is given by $n^{2}-1$ equations. Therefore $W$ has dimension at least

$$
\begin{aligned}
2 a^{2}+2 a b+2\left(8 a^{2}+6 a b+b^{2}\right) & +2(k-1) n^{2}-\left(6 a^{2}+4 a b+b^{2}-1\right) \\
-(k-1) n^{2}+k-1 & =12 a^{2}+10 a b+b^{2}+(k-1) n^{2}+k .
\end{aligned}
$$

Note that $G l_{n}(F)$ acts on $\mathcal{V}\left(\mathcal{C}_{2, n}^{(k)}\right)$ by simultaneous conjugation on each component $A_{i}$ and $B_{j}$. Let $V$ be the set of all $\left(A^{\prime}, B^{\prime}\right) \in \mathcal{V}\left(\mathcal{C}_{2, n}^{(k)}\right)$ such that $A_{0}^{\prime}$ is similar to $\lambda I+A_{0}$ for some $\lambda \in F$. Then $V$ contains the set $S=\left\{\left(\lambda+g A g^{-1}, \mu+\right.\right.$ $\left.\left.g B g^{-1}\right) \mid(A, B) \in W, \lambda, \mu \in F, g \in G l_{n}(F)\right\}$. Let us denote by $C\left(A_{0}\right)$ the centralizer of $A_{0}$ in $G l_{n}(F)$. Then $C\left(A_{0}\right)$ consists of all invertible matrices of the block form

$$
\left(\begin{array}{cccc}
X & Y & Z & P \\
0 & X & Y & 0 \\
0 & 0 & X & 0 \\
0 & 0 & Q & R
\end{array}\right) .
$$

If $\widetilde{W}$ is an irreducible component of $W$, we have a map $f_{\widetilde{W}}: G l_{n}(F) \times \widetilde{W} \times F^{2} \rightarrow$ $S \subset V \subset \mathcal{V}\left(\mathcal{C}_{2, n}^{(k)}\right)$ that takes $g,(A, B), \lambda, \mu$ to $\left(\lambda+g A g^{-1}, \mu+g B g^{-1}\right)$. For any fixed $\left(\lambda^{\prime}+g^{\prime} A^{\prime} g^{\prime-1}, \mu^{\prime}+g^{\prime} B^{\prime} g^{-1}\right)$ in the image, the fiber over this point is parameterized by the set $g^{\prime} C\left(A_{0}\right)$ and hence is of dimension equal to $\operatorname{dim} C\left(A_{0}\right)$. From this it follows that the image of $f_{\widetilde{W}}$ has dimension $n^{2}-\operatorname{dim} C\left(A_{0}\right)+\operatorname{dim} \widetilde{W}+2$, and hence the set $S$, which is the union of the images of $f_{\widetilde{W}}$ as $\widetilde{W}$ ranges through the components of $W$, has dimension $n^{2}-\operatorname{dim} C\left(A_{0}\right)+\operatorname{dim} W+2$. Thus,

$$
\begin{aligned}
\operatorname{dim} V & \geq \operatorname{dim}(S)=n^{2}-\operatorname{dim} C\left(A_{0}\right)+\operatorname{dim} W+2 \\
& \geq(3 a+b)^{2}-\left(3 a^{2}+2 a b+b^{2}\right)+12 a^{2}+10 a b+b^{2}+(k-1) n^{2}+k+2 \\
& =18 a^{2}+14 a b+b^{2}+k+2+(k-1) n^{2} .
\end{aligned}
$$

However, if $\mathcal{V}\left(\mathcal{C}_{2, n}^{(k)}\right)$ were irreducible, the expected dimension from Section 2 is $(k+1)\left(n^{2}+n\right)$, which we write as

$2\left(n^{2}+n\right)+(k-1)\left(n^{2}+n\right)=18 a^{2}+12 a b+2 b^{2}+6 a+2 b+(k-1) n^{2}+(k-1)(3 a+b)$.

Note that $\bar{V}$ is a proper subvariety of $\mathcal{V}\left(\mathcal{C}_{2, n}^{(k)}\right)$, since, for instance, the coefficient of $t^{0}$ of every element in $\bar{V}$ lies in the closed set of $M_{n}(F)$ where matrices are not 
1-regular. Hence, it suffices to find $a$ and $b$ such that

$$
\begin{aligned}
18 a^{2}+14 a b+b^{2}+k+2+(k-1) n^{2} & \\
\geq & 18 a^{2}+12 a b+2 b^{2}+6 a+2 b+(k-1) n^{2}+(k-1)(3 a+b) .
\end{aligned}
$$

This is equivalent to $b^{2}+(k+1-2 a) b+(k+1) 3 a-k-2 \leq 0$. The discriminant of this quadratic polynomial $\Delta_{k}(a)=(k+1-2 a)^{2}-4((k+1) 3 a-k-2)=4 a^{2}-$ $16(k+1) a+(k+1)^{2}+4(k+2)$ must be nonnegative, and

$$
\frac{2 a-k-1-\sqrt{\Delta_{k}(a)}}{2} \leq b \leq \frac{2 a-k-1+\sqrt{\Delta_{k}(a)}}{2} .
$$

Since $\Delta_{k}(a) \geq 0$ we get that $a \geq 2(k+1)+\frac{\sqrt{15(k+1)^{2}-4(k+2)}}{2}$ or $a \leq 2(k+1)-$ $\frac{\sqrt{15(k+1)^{2}-4(k+2)}}{2}$. Since $\sqrt{15(k+1)^{2}-4(k+2)}>3(k+1)$ for $k \geq 1$, we find in the second case that $2 a<k+1$. But the definition of $\Delta_{k}(a)$ above shows that $(k+1-2 a)^{2}>\Delta_{k}(a)$, so we find

$$
b \leq \frac{2 a-k-1+\sqrt{\Delta_{k}(a)}}{2}<\frac{2 a-k-1+|2 a-k-1|}{2}=0,
$$

which is a contradiction. So $a \geq \mu_{k}$, where we have written $\mu_{k}$ for the ceiling function $\left\lceil 2(k+1)+\frac{\sqrt{15(k+1)^{2}-4(k+2)}}{2}\right\rceil$. For each $n=3 a+b$ such that $a \geq \mu_{k}$ and $b$ satisfies the inequality (3.2) above, $V$ does not have smaller dimension than the expected dimension of $\mathcal{V}\left(\mathcal{C}_{2, n}^{(k)}\right)$, so $\mathcal{V}\left(\mathcal{C}_{2, n}^{(k)}\right)$ is reducible. In particular, for $k=1$, we find $\mu_{k}=8$, a may be taken as 8 and $b$ may be taken as 5 , so $\mathcal{V}\left(\mathcal{C}_{2, n}^{(1)}\right)$ is reducible for $n=29$.

We will now look at the solutions for $n$ for a fixed $k$ and show that for each $k \in \mathbb{N}$ there exists $N(k)$ such that $\mathcal{V}\left(\mathcal{C}_{2, n}^{(k)}\right)$ is reducible if $n \geq N(k)$. We write the inequalities in (3.2) as

$$
a-\frac{k+1}{2}-\frac{\sqrt{\Delta_{k}(a)}}{2} \leq b \leq a-\frac{k+1}{2}+\frac{\sqrt{\Delta_{k}(a)}}{2} .
$$

Adding $3 a$ to all sides, we find

$$
4 a-\frac{k+1}{2}-\frac{\sqrt{\Delta_{k}(a)}}{2} \leq 3 a+b=n \leq 4 a-\frac{k+1}{2}+\frac{\sqrt{\Delta_{k}(a)}}{2} .
$$

First, we remove the dependency of $\Delta_{k}(a)$ on $a$ by noting that if $a \geq \beta_{k}=$ $\left\lceil 2(k+1)+\frac{\sqrt{15(k+1)^{2}-4(k+1)+12}}{2}\right\rceil>\mu_{k}$, then $\sqrt{\Delta_{k}(a)} \geq 4$. Thus, we may narrow the range for $n$ given by (3.4) by choosing $a \geq \beta_{k}>\mu_{k}$ to find

$$
4 a-\frac{k+5}{2} \leq n \leq 4 a-\frac{k-3}{2} .
$$

Since the two terms on either side differ by 4 , there are four integer values starting from the smallest $n=n_{a}$, then $n_{a}+1, n_{a}+2, n_{a}+3$, that satisfy the inequality above and for which $\mathcal{V}\left(\mathcal{C}_{2, n}^{(k)}\right)$ will be reducible. Moreover, by replacing $a$ by $a+1$, we find $\mathcal{V}\left(\mathcal{C}_{2, n}^{(k)}\right)$ will be reducible for $n_{a+1}=n_{a}+4, n_{a}+5, n_{a}+6, n_{a}+7$, and so on. Hence, by taking $N(k)=\left\lceil 4 \beta_{k}-\frac{k+5}{2}\right\rceil$, we find that $\mathcal{V}\left(\mathcal{C}_{2, n}^{(k)}\right)$ will be reducible 
for $n \geq N(k)$, with $n$ written as $3 a+b$ and $a$ obtained by solving (3.5), along with the original limit of $a \geq \beta_{k}$ :

$$
\frac{1}{4}\left(n+\frac{k-3}{2}\right) \leq a \leq \frac{1}{4}\left(n+\frac{k+5}{2}\right) \text { and } a \geq \beta_{k} .
$$

(If $k=1$, we recover our earlier value of $n=29$. If $k=2$, we find $\beta_{k}=12$ and $N(2)=45$. However, working directly with (3.2), we find $\mathcal{V}\left(\mathcal{C}_{2, n}^{(k)}\right)$ is also irreducible for $n=44$ when $k=2$.)

\section{IRREDUCIBILITY OF $\mathcal{C}_{2, n}^{(k)}$ FOR $n=3$}

We will show here that when $n=3$, the set $U$ is dense in $\mathcal{V}\left(\mathcal{C}_{2,3}^{(k)}\right)$ for all $k$. This will immediately show that $\mathcal{C}_{2,3}^{(k)}$ is irreducible for all $k$. (Recall that $\mathcal{C}_{2,2}^{(k)}$ is already known to be irreducible for all $k$; see [14.)

First, we need some general reduction results that hold for any $n$. We will denote the closure of $U$ in $\mathcal{V}\left(\mathcal{C}_{2, n}^{(k)}\right)$ by $\bar{U}$.

Lemma 4.1. Assume that for all $m<n, \mathcal{V}\left(\mathcal{C}_{2, m}^{(k)}\right)$ has been proven to be irreducible. Then, any point $(A=A(t), B=B(t)) \in \mathcal{V}\left(\mathcal{C}_{2, n}^{(k)}\right)$ where $A_{0}$ or $B_{0}$ have at least two distinct eigenvalues is in $\bar{U}$.

Proof. Assume that $A_{0}$ has at least two distinct eigenvalues. Note that the eigenvalues of $A$ as an $n(k+1) \times n(k+1)$ matrix and the eigenvalues of $A_{0}$ coincide. This can be seen by writing $C=t$ in an alternative basis in the block form

$$
\left(\begin{array}{ccccc}
0 & I & 0 & 0 & 0 \\
0 & 0 & I & 0 & 0 \\
\vdots & \vdots & \vdots & \ddots & \vdots \\
0 & 0 & 0 & 0 & I \\
0 & 0 & 0 & 0 & 0
\end{array}\right)
$$

in which each entry is $n \times n$, for which correspondingly, $A$ has the form

$$
\left(\begin{array}{ccccc}
A_{0} & A_{1} & A_{2} & \ldots & A_{k} \\
0 & A_{0} & A_{1} & \ldots & A_{k-1} \\
\vdots & \vdots & \ddots & \ddots & \vdots \\
0 & 0 & \ldots & A_{0} & A_{1} \\
0 & 0 & \ldots & 0 & A_{0}
\end{array}\right)
$$

It follows from elementary considerations that since $A, B$, and $C$ commute and since $A$ has more than one eigenvalue, there exists an $H \in \mathrm{Gl}_{n(k+1)}(F)$ such that

$$
H A H^{-1}=\left(\begin{array}{cc}
P_{1} & 0 \\
0 & P_{2}
\end{array}\right), H B H^{-1}=\left(\begin{array}{cc}
Q_{1} & 0 \\
0 & Q_{2}
\end{array}\right), H C H^{-1}=\left(\begin{array}{cc}
C_{1} & 0 \\
0 & C_{2}
\end{array}\right),
$$

where the upper left block is $r \times r$ and the lower right block is $s \times s$, for some $r \geq 1$ and appropriate $s \geq 1$. Moreover, we may further adjust $H$ so that $C_{1}$ and $C_{2}$ are in Jordan canonical form. Since the Jordan form of $C$ is unique, it follows that $r=u(k+1)$ and $s=v(k+1)$ for appropriate $u$ and $v$, and $C_{1}$ consists of $u$ copies of $J_{k+1}$ while $C_{2}$ consists of $v$ copies of $J_{k+1}$. Hence, $\left(P_{1}, Q_{1}\right)$ is a point on $\mathcal{V}\left(\mathcal{C}_{2, u}^{(k)}\right)$ and $\left(P_{2}, Q_{2}\right)$ is a point on $\mathcal{V}\left(\mathcal{C}_{2, v}^{(k)}\right)$. 
We thus have a map $\mathcal{V}\left(\mathcal{C}_{2, u}^{(k)}\right) \times \mathcal{V}\left(\mathcal{C}_{2, v}^{(k)}\right) \rightarrow \mathcal{V}\left(\mathcal{C}_{2, n}^{(k)}\right)$,

$$
\left(X_{1}, Y_{1}\right),\left(X_{2}, Y_{2}\right) \mapsto\left(H^{-1}\left(\begin{array}{cc}
X_{1} & 0 \\
0 & X_{2}
\end{array}\right) H, H^{-1}\left(\begin{array}{cc}
Y_{1} & 0 \\
0 & Y_{2}
\end{array}\right) H\right)
$$

Write $Z$ for the image of this map, and note that $(A, B)$ is in $Z$ and that $Z$ is irreducible by assumption on $\mathcal{V}\left(\mathcal{C}_{2, u}^{(k)}\right)$ and $\mathcal{V}\left(\mathcal{C}_{2, v}^{(k)}\right)$. It is enough to show that $U \cap Z \neq \varnothing$ to conclude that $Z$ is contained in $\bar{U}$, since $U \cap Z$ would then be dense in $Z$. But for this, take $X_{1}=X_{1}(t)$ to be $X_{1,0}+0 t+\cdots+0 t^{k}$, where $X_{1,0}$ is a $u \times u$ diagonal matrix with distinct diagonal entries $\left(\lambda_{1}, \ldots, \lambda_{u}\right)$ and $Y_{1}=0$. Similarly take $X_{2}=X_{2}(t)$ to be $X_{2,0}+0 t+\cdots+0 t^{k}$, where $X_{2,0}$ is a $v \times v$ diagonal matrix with distinct diagonal entries $\left(\lambda_{u+1}, \ldots, \lambda_{n}\right)$, all distinct from those of $X_{1,0}$, and $Y_{2}=0$. Then, writing $H^{-1}\left(\begin{array}{cc}X_{1} & 0 \\ 0 & X_{2}\end{array}\right) H$ as $A_{0}+A_{1} t+\cdots+A_{k} t^{k}$, it is easy to see that $A_{0}$ will have the distinct eigenvalues $\left(\lambda_{1}, \ldots, \lambda_{n}\right)$, so $A_{0}$ is 1 -regular. Hence $U \cap Z$ is nonempty.

The next result is trivial but will be very useful. First, write $U^{\prime}$ for the corresponding open subset of $\mathcal{V}\left(\mathcal{C}_{2, n}^{(k)}\right)$ where $B_{0}$ is 1-regular; then $U^{\prime}$ is also irreducible by symmetric arguments. Since $U \cap U^{\prime}$ is nonempty, it follows immediately that $\bar{U}=\overline{U^{\prime}}=\overline{U \cap U^{\prime}}$.

Lemma 4.2. Let $f$ be an automorphism of $\mathcal{V}\left(\mathcal{C}_{2, n}^{(k)}\right)$ such that $f(U)=U$ or $f\left(U^{\prime}\right)=$ $U^{\prime}$ or $f\left(U \cap U^{\prime}\right)=U \cap U^{\prime}$. Then $(A, B)$ is in $\bar{U}$ iff $f(A, B)$ is in $\bar{U}$.

Since $(A, B) \mapsto(A-\lambda I, B-\mu I)$ is such an automorphism, we find:

Corollary 4.3. Let $(A, B) \in \mathcal{V}\left(\mathcal{C}_{2, n}^{(k)}\right)$ be such that $A$ has the unique eigenvalue $\lambda$ and $B$ has the unique eigenvalue $\mu$. Then $(A, B)$ is in $\bar{U}$ if and only if $(A-\lambda I$, $B-\mu I)$ is in $\bar{U}$.

As a result of Lemma 4.1 and Corollary 4.3 above, we may assume that $A$ and $B$ are nilpotent, and since the eigenvalues of $A$ and $A_{0}$ and of $B$ and $B_{0}$ coincide respectively, we may assume that $A_{0}$ and $B_{0}$ are nilpotent while proving that $(A(t), B(t)) \in \bar{U}$. We will also need the following reductions:

Corollary 4.4. Let $p(t)$ and $q(t)$ be polynomials in $F[t]$ of degree at most $k$, and assume that $q(0)=0$. Then $(A(t), B(t)) \in \bar{U}$ iff any of the following occur:

(1) $(B(t), A(t)) \in \bar{U}$,

(2) $(A(t)+p(t) I, B(t)) \in \bar{U}$,

(3) $(A(t), B(t)+p(t) I) \in \bar{U}$,

(4) $(A(t), B(t)+p(t) A(t)) \in \bar{U}$,

(5) $(A(t)(1+q(t)), B(t)) \in \bar{U}$.

Proof. For the first assertion, note that $f:(A, B) \mapsto(B, A)$ is an automorphism of $\mathcal{V}\left(\mathcal{C}_{2, n}^{(k)}\right)$ that satisfies $f\left(U \cap U^{\prime}\right)=U \cap U^{\prime}$. Thus, Lemma 4.2 applies. The second, third and fourth assertions also follow from Lemma 4.2 because the obvious maps $f$ satisfy $f(U)=U$. For the fifth assertion, for fixed $q(t)$ with $q(0)=0$, note that $1+q(t)$ is invertible in $F[t] / t^{k+1}$, with inverse $1-q^{\prime}(t)$ for some $q^{\prime}(t)$ with $q^{\prime}(0)=0$. It follows that the map $f:(A(t), B(t)) \mapsto(A(t)(1+q(t)), B(t))$ is an automorphism of $\mathcal{V}\left(\mathcal{C}_{2, n}^{(k)}\right)$ in which the coefficient of $t^{0}$ of $A(t)(1+q(t))$ is also $A_{0}$. Hence $f(U)=U$, so Lemma 4.2 applies once again. 
Finally, we have the following reduction:

Corollary 4.5. Assume that $(A(t), B(t)) \in \bar{U}$ whenever $A_{0}$ or $B_{0}$ is nonzero. Then $\bar{U}=\mathcal{V}\left(\mathcal{C}_{2, n}^{(k)}\right)$.

Proof. Take arbitrary $(A(t), B(t)) \in \mathcal{V}\left(\mathcal{C}_{2, n}^{(k)}\right)$ with $A_{0}=B_{0}=0$. First, for any $(P, Q) \in U,(\lambda P, \lambda Q)$ is also in $U$ for nonzero $\lambda \in F$; hence, taking $\lambda=0$, we find $(0,0) \in \bar{U}$. Now assume that $A(t)$ is nonzero, and assume that $A_{0}, \ldots, A_{r-1}$ are zero for some $r \geq 1$ but $A_{r}$ is nonzero. Then writing $A^{\prime}(t)$ for $A_{r}+t A_{r+1}+\cdots+t^{k-r} A_{k}$, we find $A^{\prime}$ and $A$ commute, so for nonzero $\lambda \in F,\left(A, B+\lambda A^{\prime}\right) \in \bar{U}$ by the hypothesis; so taking $\lambda=0$, we find $(A, B) \in \bar{U}$.

We now prove:

Theorem 4.6. $\mathcal{C}_{2,3}^{(k)}$ is irreducible.

Proof. It is sufficient to show that $\mathcal{V}\left(\mathcal{C}_{2,3}^{(k)}\right)$ is irreducible. For this, it is sufficient, thanks to the various reductions above and the irreducibility of $\mathcal{C}_{2,2}^{(k)}$, to show that given $(A(t), B(t)) \in \mathcal{V}\left(\mathcal{C}_{2,3}^{(k)}\right)$ with $A_{0}$ nonzero and nilpotent, the pair $(A, B)$ is in $\bar{U}$. By conjugating each of $A_{i}$ and $B_{j}$ with a fixed $H \in \mathrm{Gl}_{n}(F)$ we may assume (by Lemma 4.2) that $A_{0}$ is in Jordan form. If $A_{0}$ is 1-regular we are done, so we may assume that $A_{0}$ is the matrix $e_{1,2}$ with 1 in the $(1,2)$ slot and zeros elsewhere. We adopt the following notation for the entries:

$$
A=\left(\begin{array}{lll}
a(t) & b(t) & c(t) \\
d(t) & e(t) & f(t) \\
g(t) & h(t) & i(t)
\end{array}\right), \quad B=\left(\begin{array}{lll}
a^{\prime}(t) & b^{\prime}(t) & c^{\prime}(t) \\
d^{\prime}(t) & e^{\prime}(t) & f^{\prime}(t) \\
g^{\prime}(t) & h^{\prime}(t) & i^{\prime}(t)
\end{array}\right)
$$

We may replace $A(t)$ by $A(t)-a(t) I$ and then $B(t)$ by $B(t)-a^{\prime}(t) I$ (Corollary 4.4), so we can assume that $a(t)=a^{\prime}(t)=0$. Since $b(0)=1, b(t)$ is invertible, with the inverse of the form $1+$ higher terms, we may replace $(A(t), B(t))$ by $\left(A(t) b(t)^{-1}, B(t)\right)$ via Corollary 4.4 This does not change $A_{0}$, but sets $b(t)=1$. Next, we may replace $(A(t), B(t))$ by $\left(A(t), B(t)-b^{\prime}(t) A(t)\right)$ and assume that $b^{\prime}(t)=0$. The commutativity relation $A B=B A$ implies that

$d^{\prime}(t)=c^{\prime}(t) g(t)-c(t) g^{\prime}(t), \quad e^{\prime}(t)=c^{\prime}(t) h(t)-c(t) h^{\prime}(t), \quad f^{\prime}(t)=c^{\prime}(t) i(t)-c(t) i^{\prime}(t)$

and

$$
g^{\prime}(t)=i(t) h^{\prime}(t)-i^{\prime}(t) h(t)+c^{\prime}(t) h(t)^{2}-c(t) h(t) h^{\prime}(t)-e(t) h^{\prime}(t) .
$$

We define the matrices

$$
X=\left(\begin{array}{ccc}
0 & 0 & 0 \\
i(t)-e(t)-c(t) h(t) & 1 & 0 \\
-h(t) & 0 & 1
\end{array}\right) \quad \text { and } \quad Y=\left(\begin{array}{ccc}
0 & 0 & 0 \\
-h(t) c^{\prime}(t) & 0 & c^{\prime}(t) \\
0 & 0 & 0
\end{array}\right)
$$

Then the above equations imply that $X$ and $Y$ commute and $A Y+X B=B X+Y A$, so the matrices $A+\lambda X$ and $B+\lambda Y$ commute for each scalar $\lambda \in F$. For generic $\lambda$ the matrix $A+\lambda X$ has at least two distinct eigenvalues, so for generic $\lambda \in F$ the pair $(A+\lambda X, B+\lambda Y)$ belongs to $\bar{U}$ by Lemma 4.1 and the irreducibility of $\mathcal{C}_{2,2}^{(k)}$. Hence $(A, B) \in \bar{U}$, and we are done.

We have the immediate corollary: 
Corollary 4.7. Let $N=3(k+1)$ for $k=1, \ldots$, and let $C \in M_{N}(F)$ be of the form $\lambda I+C^{\prime}$, where $\lambda \in F$ and $C^{\prime}$ is similar to the $N \times N$ matrix consisting of three copies of $J_{k+1}$ along the diagonal. Then for any $A, B \in M_{N}(F)$ such that $A$, $B$, and $C$ all commute, we have $\operatorname{dim}_{F}(F[A, B, C]) \leq N$.

Proof. The proof is standard, given the irreducibility of $\mathcal{V}\left(\mathcal{C}_{2,3}^{(k)}\right)$. We may assume $\lambda=0$ and $C^{\prime}$ is in Jordan form since the addition of scalars and simultaneous conjugation does not change algebra dimension. On the open set $U, F[A, B, C]=F[A, C]$ by Theorem 2.2 part 4 By classical results, $\operatorname{dim}_{F}(F[A, C]) \leq N$. (In fact, by Theo$\operatorname{rem} 2.2$. part 3, $\operatorname{dim}_{F}(F[A, C])=N$ on this open set.) Since $\operatorname{dim}_{F}(F[A, B, C]) \leq N$ is a closed set condition, it holds on all of $\bar{U}$. But the nonempty open set $U$ is dense in $\mathcal{V}\left(\mathcal{C}_{2,3}^{(k)}\right)$ because of the irreducibility of $\mathcal{V}\left(\mathcal{C}_{2,3}^{(k)}\right)$. Hence $\bar{U}=\mathcal{V}\left(\mathcal{C}_{2,3}^{(k)}\right)$, and the theorem follows.

\section{A special Result when $\operatorname{dim}_{F}\left(F\left[A_{0}, B_{0}\right]\right)=n$}

We may combine the ideas in $\S 2.2$ and in the proofs of Theorem 2.2 and Corollary 4.7 to establish the following:

Proposition 5.1. Let $A(t)=A_{0}+A_{1} t+\cdots+A_{k} t^{k}$ and $B(t)=B_{0}+B_{1} t+\cdots+$ $B_{k} t^{k}$ commute in $M_{n}(F)[t] / t^{k+1}$, and assume that $\operatorname{dim}_{F}\left(F\left[A_{0}, B_{0}\right]\right)=n$. Then viewing $M_{n}(F)[t] / t^{k+1} \subset M_{n(k+1)}(F)$ as usual with $t$ corresponding to the matrix $C$ consisting of $n$ copies of $J_{k+1}$ along the diagonal, we have $\operatorname{dim}_{F}(F[A, B, C])=$ $n(k+1)$.

Proof. The same arguments as in $\S 2.2$ applied to the entire open subscheme of $\mathcal{C}_{2, n}$ consisting of regular points show that the open algebraic set $Y$ in $\mathcal{V}\left(\mathcal{C}_{2, n}^{(k)}\right)$ where $\operatorname{dim}_{F}\left(F\left[A_{0}, B_{0}\right]\right)=n$ is irreducible. Since $Y \supset U$ and $Y$ is irreducible, the closure of $Y$ equals $\bar{U}$. We have seen in the proof of Corollary 4.7 that $\operatorname{dim}_{F}(F[A, B, C]) \leq n(k+1)$ on $\bar{U}$; thus, this relation holds on the closure of $Y$. Now, as in the proof of Theorem $2.2((3) \Rightarrow(1))$, we may define $E_{i}$ to be the $F$-subspace of $F[A, B, t] \subset M_{n}(F)[t] / t^{k+1}$ of elements of degree at least $i$. We then have injective $F$-space maps $E_{i} / E_{i+1} \stackrel{\cdot t}{\rightarrow} E_{i+1} / E_{i+2}$, which coupled with the fact that $\operatorname{dim}_{F}\left(E_{0} / E_{1}\right)=\operatorname{dim}_{F}\left(F\left[A_{0}, B_{0}\right]\right)=n$, show that $\operatorname{dim}_{F}(F[A, B, C])=$ $\sum_{i=0}^{k} \operatorname{dim}_{F}\left(E_{i} / E_{i+1}\right) \geq n(k+1)$. Equality now follows.

\section{REFERENCES}

1. F.R. Gantmacher, The Theory of Matrices, Vol. 1, Chelsea Publishing Company, New York, 1959. MR 0107649(21:6372c)

2. M. Gerstenhaber, On dominance and varieties of commuting matrices, Annals of Mathematics (2), 73 (1961), 324-348. MR0132079 (24:A1926)

3. Russell Goward and Karen Smith, The jet scheme of a monomial scheme. Comm. Algebra, 34 (2006), 1591-1598. MR 2229478 (2007e:13037)

4. R. Guralnick, A note on commuting pairs of matrices, Linear and Multilinear Algebra, 31 (1992), 71-75. MR1199042 (94c:15021)

5. Boyan Jonov, Shellability of a complex associated to the first order jet scheme of a determinantal variety, in preparation.

6. Tomaž Košir and B.A. Sethuraman, Determinantal varieties over truncated polynomial rings, Journal of Pure and Applied Algebra, 195 (2005), 75-95. MR2100311 (2005h:13020)

7. Tomaž Košir and B.A. Sethuraman, A Groebner basis for the $2 \times 2$ determinantal ideal $\bmod t^{2}$, J. Algebra, 292 (2005), 138-153. MR2166800 (2006f:13011)

8. Qing Liu, Algebraic Geometry and Arithmetic Curves, Oxford Grad. Texts Math., 6, Oxford University Press, 2002. MR,1917232 (2003g:14001) 
9. Mircea Mustaţă. Jet schemes of locally complete intersection canonical singularities. Invent. Math., 145 (3) (2001), 397-424. MR.1856396 (2002f:14005)

10. Mircea Mustaţă. Singularities of pairs via jet schemes. J. Amer. Math. Soc., 15(3) (2002), 599-615. MR 1896234 (2003b:14005)

11. T. Motzkin and O. Taussky-Todd, Pairs of matrices with property L. II, Trans. Amer. Math. Soc., 80 (1955), 387-401. MR0086781(19:242c)

12. John F. Nash, Jr., Arc structure of singularities. A celebration of John F. Nash, Jr., Duke Math. J., 81 (1995) (1), 31-38 (1996). MR1381967 (98f:14011)

13. Michael G. Neubauer and David J. Saltman, Two-generated commutative subalgebras of $M_{n}(F)$, J. Algebra, 164 (1994), 545-562. MR1271255 (95g:16030)

14. M.J. Neubauer and B.A. Sethuraman, Commuting pairs in the centralizers of 2-regular matrices, Journal of Algebra, 214 (1999), 174-181. MR.1684884 (2000h:15024)

15. Cornelia Yuen, Jet Schemes and Truncated Wedge Schemes, Ph.D. thesis, University of Michigan (2006).

Department of Mathematics, California State University, Northridge, Northridge, CALIFORNIA 91330

E-mail address: al.sethuraman@csun.edu

Institute of Mathematics, Physics, and Mechanics, University of Ljubljana, JadranSKa 19, 1000 Ljubluana, Slovenia

E-mail address: klemen.sivic@fmf.uni-lj.si 